\title{
Plädoyer für eine modulare Psychotherapie
}

\author{
Fritz Hohagen Winfried Lotz-Rambaldi \\ Zentrum für Integrative Psychiatrie ZIP gGmbH, Klinik für Psychiatrie und Psychotherapie Lübeck, Deutschland
}

Psychotherapie entwickelt sich stetig weiter - und damit auch die Verhaltenstherapie. Ihre in Wellen verlaufende Entwicklungsgeschichte wurde in den letzten 60 Jahren entsprechend ihrem Selbstverständnis vor allem von empirischen Befunden geprägt, aber auch immer wieder von verschiedenen wissenschaftstheoretischen und philosophischen Menschenbildern beeinflusst. Diese Weiterentwicklung führte dazu, dass die Verhaltenstherapie längst als dominierendes Richtlinienverfahren fest im deutschen Gesundheitssystem verankert ist. Die Kognitive Verhaltenstherapie stellt seit Langem das bestevaluierte Psychotherapieverfahren dar, und auch ihre diversen indikationsspezifischen Ausgestaltungen und Weiterentwicklungen sind einer empirischen Absicherung verpflichtet, wenngleich kritisch festgestellt werden muss, dass manche Grundannahmen und Interventionen, die der dritten Entwicklungswelle der Verhaltenstherapie zugrunde liegen, einer empirischen Überprüfbarkeit nur noch schwer zugänglich sind.

Die immer stärker spezialisierten und zunehmend «manualisierten» Therapieverfahren innerhalb der Verhaltenstherapie sahen sich allerdings schon in den 1990er Jahren dem Vorwurf von Kritikern ausgesetzt, als Manualgestützte Psychotherapie lediglich eine «Therapie von der Stange» anzubieten, die einer individuellen Behandlung unterlegen sei. Die seit dieser Zeit immer stärkere enorme Ausdifferenzierung innerhalb der Verhaltenstherapie und ihre vielfältigen Weiterentwicklungen sowie die damit einhergehende zunehmende Fragmentierung und drohende «Kleinstaaterei» der Verhaltenstherapie und ihrer Protagonisten wurde bereits mehrfach auch an dieser Stelle kritisch kommentiert [Ehlert, 2009; Hohagen und Lotz-Rambaldi, 2011]. Heute kann kein Psychotherapeut mehr alle Manuale lernen und beherrschen, die für mehr als 100 Störungsbilder und Problembereiche entwickelt wurden. Weiter wurde kritisiert, dass Therapie-Manuale, die für ein bestimmtes
Krankheitsbild entwickelt wurden, dem Problem der Multikomorbidität unserer Patienten nicht gerecht werden. Außerdem lassen sich neuere psychotherapeutische Methoden immer weniger eindeutig bisherigen Therapieverfahren und -schulen zuordnen, da sich ihre Konzepte aus verschiedenen Theorien und Traditionen speisen. Die Vielzahl neuer Methoden auf dem verhaltenstherapeutischen Markt macht es selbst für psychologische und ärztliche Psychotherapeuten mit einer fundierten Verhaltenstherapieausbildung zunehmend unmöglich, diese neuen Methoden z.B. Cognitive Behavioral Analysis System of Psychotherapy (CBASP), Schematherapie, Dialektisch-Behaviorale Therapie (DBT) - und ihre Verzweigungen in Zusatzausbildungen $\mathrm{zu}$ erlernen und sie nach entsprechender Qualifizierung und Erfahrung auch selbst an andere, z.B. im Rahmen der psychiatrisch-psychotherapeutischen Weiterbildung, weiterzugeben.

In den Fachgesellschaften verläuft daher seit geraumer Zeit ein Diskussionsprozess darüber, ob eine alternative Herangehensweise in der Weiterbildung notwendig ist bzw. wie diese sinnvoll umgesetzt werde kann. In Arbeitsgruppen der Deutschen Gesellschaft für Psychiatrie, Psychotherapie und Nervenheilkunde (DGPPN) wurden hierzu konkrete Überlegungen angestellt. Eine künftige, veränderte Weiterbildung könnte demnach - bezogen auf den psychotherapeutischen Behandlungsbereich, der in jedem Fall als fester Bestandteil der Psychiatrie zu begreifen ist - in einer Art Modulsystem organisiert sein, in dem bestimmte therapeutische Grundfertigkeiten und Kompetenzen wie kognitive Umstrukturierung, soziales Kompetenz- oder Problemlösetraining, aber auch Emotionsregulation, Achtsamkeitsfokussierung, Ressourcen- und Klärungsorientierung usw. vermittelt werden. Dieses neu zu schaffende Baukastensystem würde es dann - jenseits des «Schulenstreits» - erlauben, sich möglichst früh wichtige Basisfertigkeiten im Rahmen eines

\section{KARGER}

Fax +497614520714

Information@Karger.de

www.karger.com (c) 2012 S. Karger GmbH, Freiburg

$1016-6262 / 12 / 0222-0084 \$ 38.00 / 0$

Accessible online at:

www.karger.com/ver
Prof. Dr. Fritz Hohagen

Zentrum für Integrative Psychiatrie ZIP gGmbH

Klinik für Psychiatrie und Psychotherapie

Ratzeburger Allee 160, 23538 Lübeck, Deutschland

Tel. +49 451 5002-440/1, Fax -603

fritz.hohagen@uksh.de 
Grund-Curriculums (1. und 2. Weiterbildungsjahr) anzueignen. Im Rahmen eines Aufbau-Curriculums (3. und 4. Weiterbildungsjahr) würden dann daran anschließend neue, störungsspezifische und evidenzbasierte psychotherapeutische Methoden bzw. Richtlinienverfahren mit weniger Zeitaufwand als bisher erlernt und diese in die bereits vorhandenen Behandlungskonzepte integriert werden.

Der Psychotherapeut, der eine solche modulare Aus- und Weiterbildung in der Psychotherapie durchlaufen hat, kann dann, individuell auf die jeweiligen Bedürfnisse des Patienten angepasst, ein flexibles Behandlungskonzept anwenden, das den einzelnen Problembereichen innerhalb eines oder mehrerer Krankheitsbilder besser Rechnung trägt. Dieses Modell wäre zudem gleichermaßen für den Instituts- wie auch für den Ausbildungsbereich geeignet.

\section{Literatur}

Ehlert U: Weiterentwicklungen in der Verhaltenstherapie: Unübersehbar und identitätszerstörend? Verhaltenstherapie 2009;19:216-217.

Hohagen F, Lotz-Rambaldi W: Zur Fragmentierung in der Verhaltenstherapie. Verhaltenstherapie 2011;21:84-85. 